\title{
Gene Editing and Genome Engineering with CRISPR-Cas9*
}

\author{
Emmanuelle Charpentier ${ }^{1}$
}

\begin{abstract}
The RNA-programmable CRISPR-Cas9 technology allows precise and efficient engineering or correction of mutations, modulation of gene expression and marking of DNA in a wide variety of cell types and organisms in the three domains of life. Because of its versatility and ease of design, this powerful technology has been rapidly and universally adopted for genome editing applications in life science research. It is also recognized for its promising and potentially transformative applications in biotechnology, medicine and agriculture.
\end{abstract}

Keywords: CRISPR-Cas9; Gene Editing; Genome Engineering; Designer Nucleases; Streptococcus pyogenes; tracrRNA.

Emmanuelle Charpentier: Thank you for the kind introduction. CRISPR-Cas9 is about mobile genetic elements in bacteria, and indeed to understand mobile genetic elements during my career, it has been helpful to be mobile myself as a big human mobile genetic element! Thank you, Jennifer, for giving some introduction about CRISPR-Cas systems. I also would like to thank the Molecular Frontiers Foundation, Bengt Nordén and the Royal Swedish Academy of Sciences for hosting this event. I think it is essential in our days to promote awareness of chemistry, science, and biology for young audiences like you. I will speak about the background of CRISPR-Cas9 and the applications of this technology. I just want to remind you that CRISPR-Cas9 is about this enzyme, Cas9 - here represented as scissors - that can be programmed with RNA molecules to target any DNA sequence. The RNA components work as a kind of GPS system to guide Cas9 to any part of the genome in any cell or organism. So it is a very powerful technology. Among all the different CRISPR-Cas systems existing, the CRISPR-Cas9 system is also quite minimal. And indeed it is the simplicity yet sophistication of this technology that have made it a valuable tool for programmable genome engineering.
The explosion of interest in the CRISPR technology has been called the CRISPR Craze. This was highlighted by Elizabeth Pennisi for the journal Science in the summer of 2013, nearly four years ago, and already coined as the CRISPR Craze with the subtitle "A bacterial immune system yields a potentially revolutionary genome-editing technique". And this is really about this technology that allows precise gene surgery in any cell or organism. This article was published only a year after we published with Jennifer Doudna the article in Science in June 2012 explaining the last step of the CRISPR-Cas9 mechanism and how to harness this mechanism as a powerful technology for genetics. This shows how quickly and easily the technology was adopted by the scientific community.

And very fast as well, the CRISPR-Cas9 field has been highlighted in the media, first as the next medical breakthrough, but then recognizing as well the potential of the technology in other fields of life sciences and agriculture, as was mentioned by Jennifer Doudna earlier. Surely, a number of scientific articles were published following up on the CRISPRCas9 mechanism, including enhancements of the technology and all kinds of applications. So CRISPR-Cas9 has already

\footnotetext{
${ }^{1}$ Max Planck Institute for Infection Biology, 10117 Berlin, Germany. Published online 21 November 2017; doi:10.1142/S2529732517400119 *This article was transcribed from a presentation delivered by Professor Charpentier at the Molecular Frontiers Symposium on Tailored Biology at the Royal Swedish Academy of Sciences, May 2017. It is published here in MFJ with permission from Professor Charpentier. This is an Open Access article published by World Scientific Publishing Company. It is distributed under the terms of the Creative Commons Attribution 4.0 (CC-BY) License. Further distribution of this work is permitted, provided the original work is properly cited.
} 


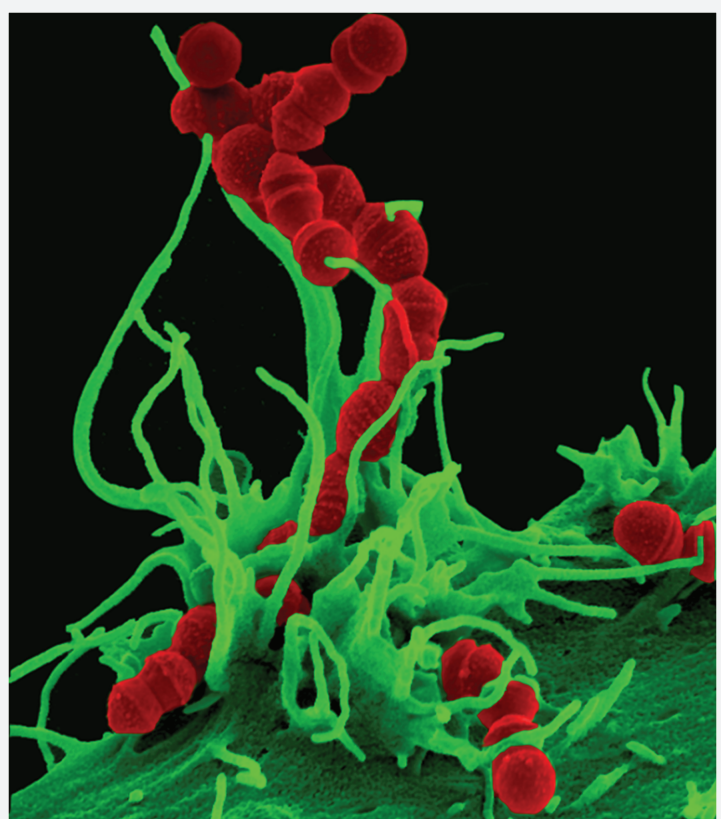

The Charpentier Lab studies the biology of pathogens, with particular focus on the mechanisms and regulation of bacterial infection. This electron micrograph shows the human pathogen Streptococcus pyogenes infecting human epithelial cells. However, bacteria can themselves succumb to infection with bacteriophages and other mobile genetic elements. To defend themselves against such infections, they have evolved a variety of defense mechanism, including CRISPR-Cas. Image credit: Manfred Rohde (Helmholtz Centre for Infection Research, Braunschweig, Germany).

been very helpful as a technology for the field of life sciences and it will still remain very helpful in the years to come.

My initial interest in CRISPR-Cas9 came from my desire to understand diseases at the fundamental level. I belong to those scientists who are interested in the molecular and cellular mechanisms behind the diseases. A number of my colleagues are focused on cancer, or on cardiovascular, neurological or genetic diseases. And I belong to those people interested in infectious diseases caused by bacteria. Bacterial pathogens that cause disease in humans have been the focus of my career over the last 25 years. And we always hope, when we do fundamental work on different types of cells and organisms, that we will identify pathways, genes and molecules that allow us to really understand the functions of genes and understand all the molecular and cellular mechanisms of life. But we also do this research hoping that maybe we will find an interesting mechanism that could be harnessed for novel therapies or novel technologies. And having worked on bacteria for a long time and having been trained at the Pasteur Institute, I always had this notion that it was very important to focus on basic science, as this was an excellent way to find interesting mechanisms that could be harnessed, in the case of bacterial diseases, to find new antibiotics that can kill bacteria, but also to discover or improve genetic tools. Indeed, bacteria and viruses are the sources of multiple enzymes that have been used in molecular biology and genetics over the last decades.

In my laboratory, we not only work on CRISPR-Cas9, but also on RNA- and protein-mediated regulatory mechanisms that allow us to understand how bacteria survive in their environment and how they can cause diseases. We always have in mind that it is critical to continue to work on bacterial infectious diseases since it is the leading cause of death in children and adolescents worldwide, and since antibacterial resistance is still a major health threat, as highlighted recently by the United Nations. Now having said this, genetics is really critical in laboratories for understanding functions of genes, and with regard to the medical field, for example, to find new targets for therapeutics. And I just would like to give you an idea of where CRISPR-Cas9 can be placed in the history of genetics towards gene editing. And surely, I mention only selected breakthroughs. The nineteenth century has been very critical for fundamental genetics and for establishing the rules of genetics - with Darwin's On the Origin of Species, Mendel on the segregation of alleles and Friedrich Miescher who isolated DNA in 1871. Then in the middle of the last century, it was shown that DNA was the carrier of genetic information, the double helix structure of DNA was determined, and the genetic code was revealed. This was very exciting, and with the possibility to really understand the code of genes, it also became necessary to develop technologies that would allow one to target genes and understand their expression and functions. This really started in the ' 60 s and ' 70 s with a number of scientists interested in working with bacteria, which were at the time the main organisms that one could work with genetically. Here, I would like to mention a quote from Jacques Monod, a French microbiologist working at the Pasteur Institute. He, together with François Jacob and André Lwoff, received the Nobel Prize in 1965 for their very interesting concepts on proteins with enzymatic properties in bacteria, the notion of genes organized as operons, the notion of RNA molecules that could have regulatory functions beyong being the intermediate between the DNA and proteins. This research - having been done in bacteria - was also trying to understand how bacteria defend themselves against viruses, in this case lysogenic phages. I just want to mention this because this is exactly what CRISPR-Cas9 is about: it is an RNA- and protein-mediated system in bacteria, and it is about interactions with bacteriophages. This shows that 40 to 50 years later you can take interesting breakthroughs that were highlighted in the past, and dig into the details of topics which may not sound that exciting or fashionable, but you can still find very interesting points. Although Jacques Monod was very visionary, in 1970 he wrote a book entitled Chance and 


\section{Finding small regulatory RNAs in S. pyogenes}

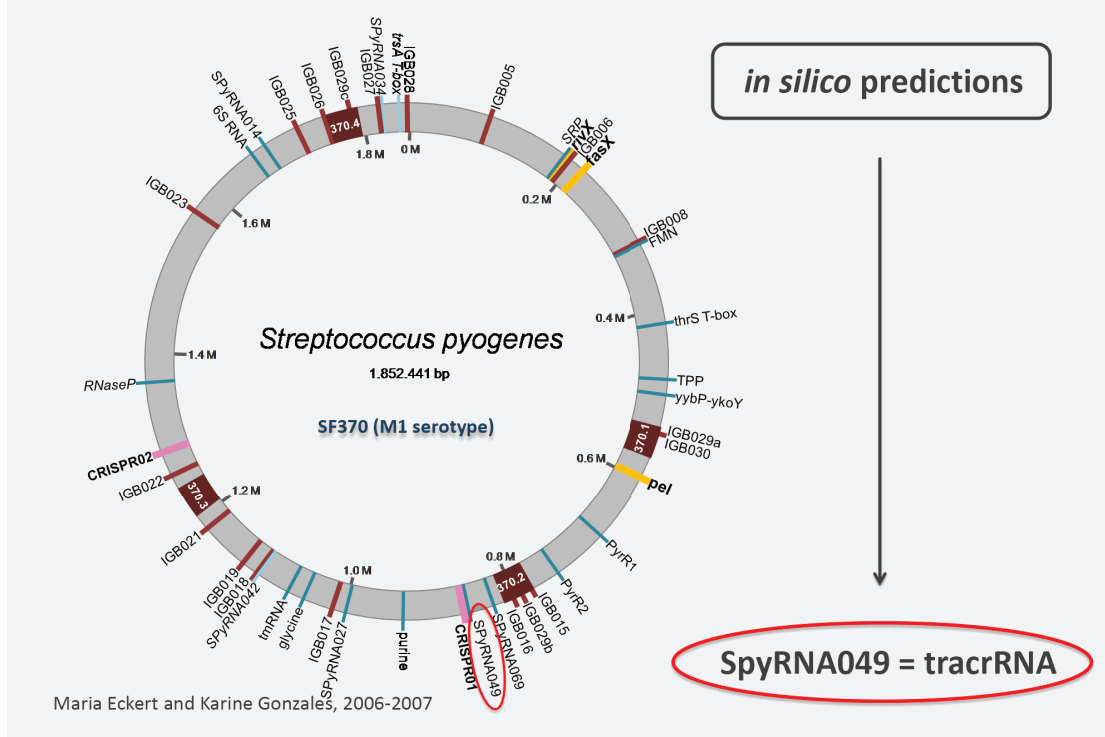

Investigation of small regulatory RNAs in S. pyogenes led to the discovery of trans-activating CRISPR RNA (tracrRNA). manner. Zinc finger nucleases were developed in the '90s, and TALEN nucleases later, in 2010. These types of engineered proteins came from research on meganucleases and homing nucleases in eukaryotic systems. Some scientists did really wonderful work to engineer those nucleases in a way that they contain a code to be able to recognize a certain sequence of interest on the DNA. The idea is that the scientist engineers a new protein with a certain code to recognize a certain sequence of DNA of interest. However, protein engineering is not always that fast and easy for biologists in the lab. So these enzymes were very good at introducing changes, but time-consuming to design and produce, and difficult to use in a democratic fashion. This is really what CRISPR-Cas9 brings, because its programmability is
Necessity where he said "Modern molecular genetics offers us no means whatsoever for acting upon the ancestral heritage so as to improve it with new features - to create a genetic 'superman'; on the contrary, it reveals the vanity of any such hope: the genome's microscopic proportions today and probably forever rule out manipulation of this sort." Unfortunately, he died at a relatively early age, but I am sure he would be very glad to see and to hear about CRISPR-Cas 9 - it is about exactly what he was studying. I would also like to mention François Jacob, who said "Nature is a tinkerer, not an inventor." Actually, if you look at what every cell and organism are doing, specifically bacteria, they are just doing bricolage, or tinkering. As scientists, we are just here to try to understand the tricky tinkering of bacteria and other organisms so I think that it is also a very interesting quote.

Having said this, the basics for the genetic tools really were developed the mid-'70s with the discovery of a number of enzymes originating from bacteria also from viruses, particularly from studies trying to understand how bacteria defend themselves against mobile genetic elements. These restriction enzymes allowed one to recombine DNA. The ability to sequence DNA and the ability to amplify DNA were also developed during this time. On this slide, I have not mentioned the RNA interference mechanisms that were developed into technologies in the beginning of 2000s, to really target gene expression. The ability to do genetics in a precise manner is the field of gene editing, which is done with engineered nucleases that have the ability to target DNA in a sequence-specific brought by the RNA component, which can be easily designed and produced.

There is a very interesting story in this field of research, and I will mention a number of scientists who have worked on CRISPR-Cas. In my lab, the interest in CRISPR-Cas9 came really from the focus of my research when I started my independent career as a principal investigator. We were working specifically on Streptococcus pyogenes, which is a human pathogen responsible for a large number of diseases from mild infections to more severe infections. Here you can see the cocci, which are coloured in red for the purpose of the picture, and they are invading human epithelial cells - pharyngeal cells - coloured in green for the purpose of the picture. We are interested in different types of mechanisms - both RNAand protein-based - underlying how bacterial pathogens attack the human host and, to a certain extent, how the human host can defend itself against bacterial pathogens. We focus on protein quality control, the class of small regulatory RNAs and other regulatory mechanisms that allow us to understand how virulence factors and other factors allow the bacteria to react very fast to their dynamic and diverse environments. The constant challenge of rapidly adapting to their environment and here I would like to refer to the talk of Frances Arnold this morning - has led to the evolution of an immense variety of proteins and mechanisms in the bacterial world. The CRISPRCas9 system arose from constant battle of the bacteria with bacteriophages. But bacteria encounter other types of environments and stresses, specifically those in the human host 
Type II CRISPR-Cas: model of crRNA maturation

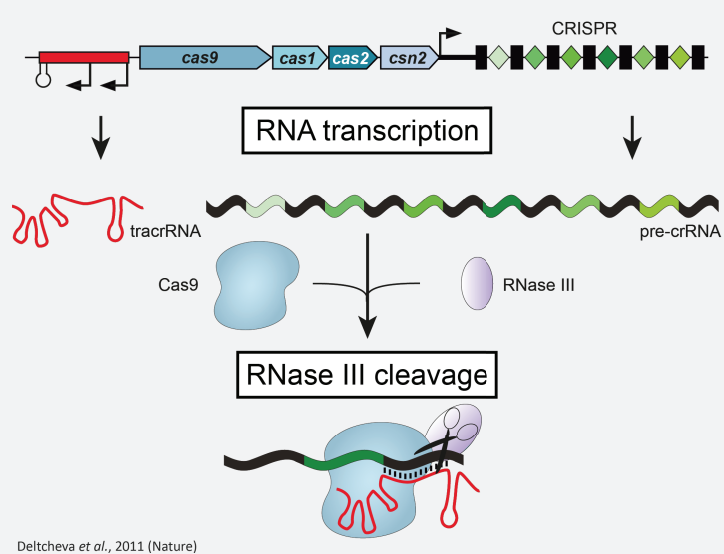

tracrRNA bears complementarity to the repeats in crRNA, and directs the RNase III-mediate maturation of crRNA when the two RNAs are bound to the protein Cas9. This is a critical step in CRISPR-mediated immunity to invading nucleic acids. The deep molecular understanding of tracrRNA-mediated mechanisms revealed the basic molecular principle of DNA targeting by CRISPR-Cas 9 and a means to repurpose this mechanism as a RNA-programmable genetic tool.

such as osmotic stress, pH changes, heat shock and the host immune response.

We are very much interested in everything that is the dynamic of regulation of gene expression in bacteria. We became interested in the family of small RNAs a little bit more than 15 years ago. The notion, which Jennifer Doudna explained to you earlier, is that RNA — in addition to being the intermediate molecule between DNA and protein — can also have other functions. You also have RNA molecules that have regulatory function and do not code for any peptide or protein. And in the bacterial world, this field of research is quite exciting. A large number of smaller regulatory RNAs have been identified, mostly in Gram-negative bacteria. What was found is that they use a multitude of different mechanisms to affect gene expression. For instance, they can directly interact with messenger RNA forming antisense structures. By this interaction with the messenger RNAs that code for proteins, they can either enhance or inhibit the stability of the messenger RNA. They can also activate or inhibit the translation via different means. They can also interact directly with proteins to activate or sequester them. When we started to work on CRISPR and small RNAs, there were types of mechanisms that were not yet described in bacteria, namely small RNAs that might activate other small RNAs through a direct interaction, and small RNAs that might interact directly with DNA and affect the fate of the DNA. This is really what CRISPR-Cas biology ended up bringing in terms of a new concept, a new dogma. We were interested in finding novel, RNA-based mechanisms in Streptococcus pyogenes.

I am just going to give you a little bit of background on how we came to CRISPR-Cas9, and then I will go very fast. In principle, we were working in my lab on some small regulatory RNAs for which we found a biological function in influencing the potential of the bacterium to cause diseases by directly regulating the expression of genes coding for virulence factors. And a little bit more than 10 years ago we were interested in finding new, small regulatory RNAs in Streptococcus pyogenes. We approached the problem by using bioinformatics, which allowed us to identify a number of sequences in the genome of Streptococcus pyogenes that potentially could code for RNA molecules that might have interesting biological functions. And we focused on a molecule that we called at the time SpyRNA049, but later renamed tracrRNA. What we found is that this RNA molecule was actually encoded in the vicinity of a gene in the genome of Streptococcus pyogenes that was already highlighted at the time as a gene encoding a CRISPR system-related protein containing two nuclease domains. This protein was previously called Csn1, but was renamed Cas9 when the standard CRISPR nomenclature was agreed upon. This is really about this protein, Cas9, that indeed contains two nuclease domains to cleave the DNA.

We were focusing mainly on tracrRNA as an RNA molecule with a very interesting expression pattern, being abundant and expressed in three different forms. We were also interested in CRISPR because we thought that it could have a role in limiting the acquisition of the viruses carrying genes that promote the virulence of Streptococcus pyogenes. Indeed, we could ultimately show that in this bacterium CRISPR can limit the acquisition of genes that are important for the severe aspects of Streptococcus pyogenes infections through limiting the acquisition of these viral genomes. So in principle we were also interested in CRISPR immune system, but we were initially working on this tracrRNA molecule and on CRISPR RNA (crRNA) molecules independently. But it came to the point where it was important to look at the interaction of those two components.

Having said this, CRISPR really brought us to another type of infection biology where the bacteria are not pathogens of the human host, but the bacteria can be host themselves and they can be attacked by these mobile genetic elements. I mentioned viruses, but you also have plasmids that can bring very interesting genes to the bacteria, allowing the bacteria to resist the action of antibiotics or to adapt better to their environment. And sure enough, the bacteria have evolved defence systems against those mobile genetic elements. Here for example, you have a phage infecting a bacterial cell. Some phages can enter the so-called lytic cycle, where the genome of the phage is injected into the bacterial cell, replicates, and produces new phage particles. These will kill the bacterial 
Type II CRISPR-Cas: model of DNA targeting

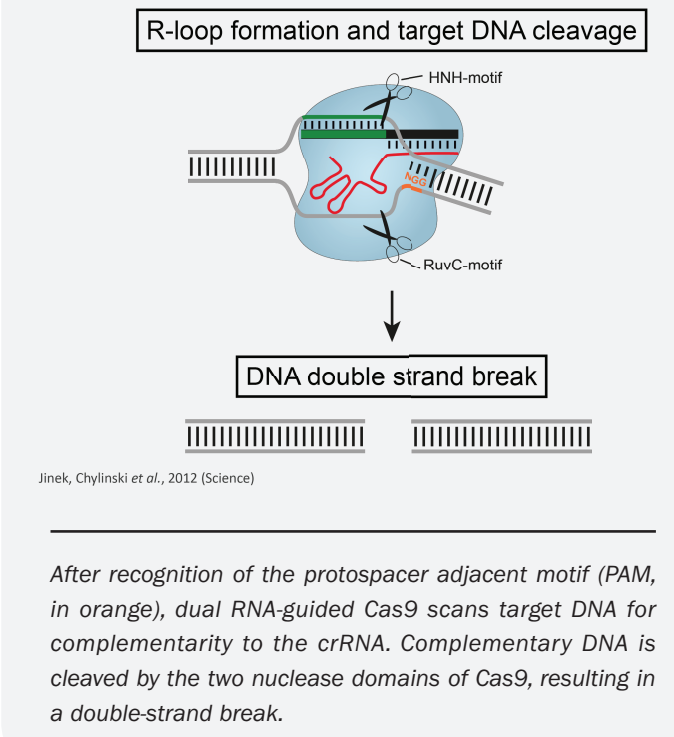

cell, releasing more phages to kill other bacterial cells. And here, you have the case where the phage can enter the socalled lysogenic cycle. These temperate phages insert their genomes as prophages into the genome of the bacteria. In this case, the viruses bring new genetic traits to the bacteria. But in either case, and also when bacteria are attacked by plasmids or transposons, they have defence systems that allow them to attack these mobile genetic elements. There are different types of defence systems, most of which are considered innate immunity, in reference to innate and adaptive immunity in eukaryotic organisms. Restriction enzymes are used to cleave DNA and clone DNA, and were identified by understanding bacterial viral defence mechanisms against viruses. In addition to restriction-modification systems, you have other types of innate immunity in bacteria. CRISPR-Cas is the first and so far the only adaptive immune system described in prokaryotes, existing not only in bacteria but also in archaea. It is adaptive because you first have recognition and memorization of the mobile genetic element, which then protects against subsequent attack by the same mobile genetic element.

Jennifer already explained that the system contains a CRISPR array, which is composed of short sequences - here shown in black - that are identical to one another. These are called repeats, and they are interspaced by so-called spacer sequences that originate from mobile genetic elements. This CRISPR array contains a region upstream of the array that allows this array to be transcribed into a long RNA molecule. And in the vicinity of this CRISPR array, you have a series of genes that code for the protein components. In principle, you have the proteins and you have the crRNAs. You have three steps in the immune system: spacer acquisition, expression of the crRNAs, and then interference, whereby the system recognizes and destroys a mobile genetic element.

CRISPR-associated proteins recognize the invading DNA and insert it into the leader-proximal side of the CRISPR array. This provides a memory of the initial infection with the mobile genetic element. This is a series of spacers, which represent memorized infections. This array is transcribed to produce a long precursor crRNA molecule, which then undergoes maturation, meaning that it is cleaved to yield individual crRNAs that can guide a complex of CRISPR-associated proteins to the cognate viral genome. This is accomplished by base pairing of the crRNA with the complementary sequence in the invading viral genome. UItimately, the complex will recognize this interaction and will cleave the viral DNA to prevent viral propagation or maintenance.

There is a very interesting story in the field of the CRISPR-Cas biology that I briefly want to mention because it is important that you realize that there are surely always a lot of scientists involved, each bringing different pieces of the puzzle. Thirty years ago, a Japanese group identified this series of repeats in the genome of Escherichia coli, but they did not know what the function of these repeats was. And then at the end of the ' 90 s and in the early 2000s, some scientists showed using so-called Northern blot analysis that the CRISPR array could produce RNA molecules of different sizes. They therefore thought that RNA cleavage was occuring. And then in the early- and mid-2000s, bioinformaticians found that in the vicinity of the series of repeats, you had genes encoding the CRISPR-associated proteins with interesting features. These proteins were similar to other proteins that can interact with DNA and RNA, or cleave DNA and RNA. And then what was also found is that these series of repeats were interspaced by sequences that seem to always match sequences of viruses, plasmids, and transposons. As Jennifer mentioned, the idea was that this could represent an interference system in bacteria and archaea that would be RNA-mediated, composed of RNA-protein complexes, and allow the bacteria and archaea to fight the invasion of mobile genetic elements. Surely, I have omitted a lot of studies, but the group of Horvath and Moineau with Barrangou showed in 2007, working on Streptococcus thermophilus, that CRISPRCas was indeed an adaptive immune system in bacteria. A number of colleagues described other CRISPR-Cas systems. Multiple types have evolved and they are mainly classified based on the composition and nature of their CRISPR-associated genes. Stan Brouns and John van der 0ost showed that the type I system could target DNA, and Terns, Marraffini and Sontheimer described the type III systems. These types had different mechanisms, but always used one crRNA molecule and a complex of CRISPR-associated proteins. Surely, I have 


\section{CRISPR-Cas9: RNA-programmable DNA cleavage}

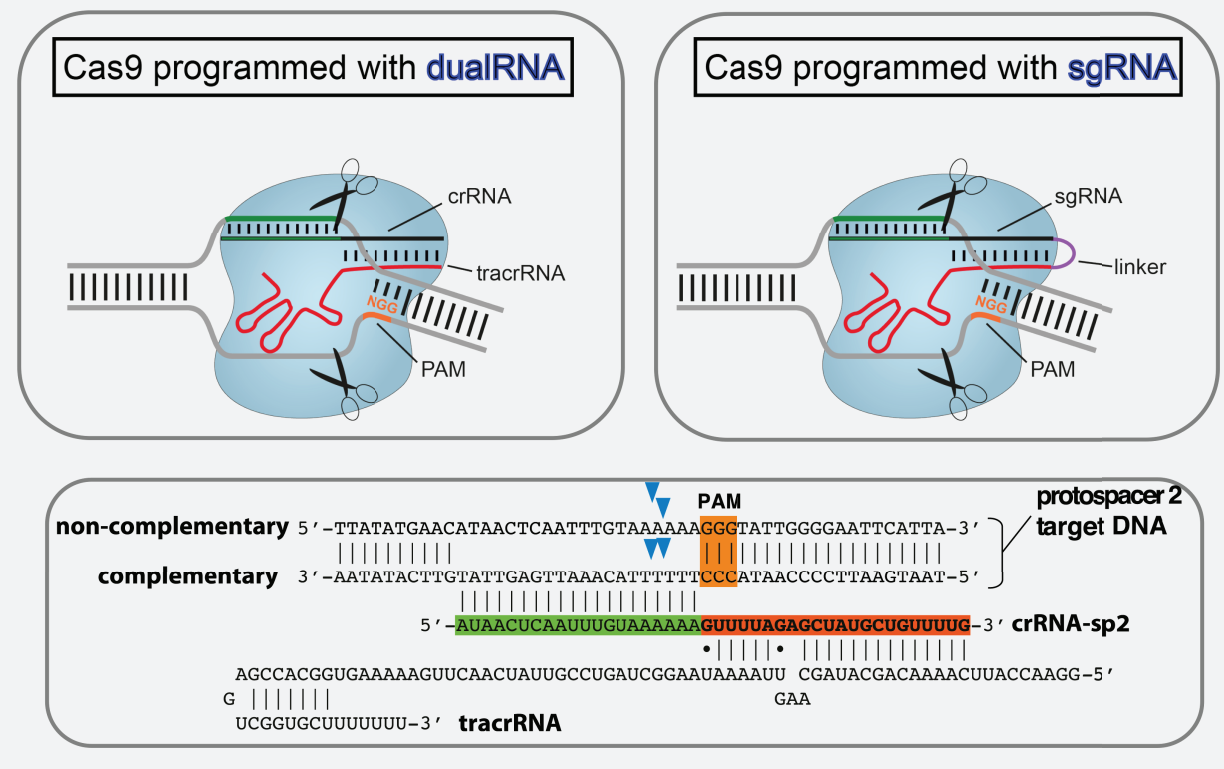

Jinek, Chylinski et al., 2012 (Science)

Cas9 can be programmed with dual RNA (crRNA and tracrRNA) or with a single guide RNA (sgRNA) that combines features of both crRNA and tracrRNA.

omitted important contributions of a number of scientists in the last 10 years. But what is really peculiar with the type II CRISPR-Cas9 system is its simplicity - it was different from the type I and type III system that were being studied when we became interested in CRISPR-Cas.

CRISPR-Cas9 is unique because instead of a complex of proteins targeting the genomes, you have only one protein involved, Cas9. And instead of one RNA molecule that contains the memorized mark of the mobile genetic element, you have two RNA molecules. We initially thought that tracrRNA, the second RNA, was involved in the regulation of expression of a virulence factor. We had found a very interesting target, but we could not make any sense of this interaction. Then the idea that I had was that maybe tracrRNA would have an active role in the CRISPR-Cas9 system, because at the time we did not know anything about the regulation of the system, and also because when you work in the RNA field, you know that some RNA molecules are encoded next to their target, and can work in cis or in trans. And then we started to decipher the mechanism, showing that the three components of the system were Cas9, tracrRNA and crRNA. The duplex of RNA stabilized by Cas 9 allows production of the mature crRNAs that can guide Cas9 protein to the target genome.

We were not only working on Streptococcus pyogenes. Actually, very early on, we thought it was important to understand the diversity of the system in the bacterial world. And it is by looking at the evolution of the system that we understood the rules of how the components could interact with one another. By looking at multiple bacterial species we in my laboratory understood very early on that the sequences of tracrRNA, crRNA and the Cas9 protein were extremely diverse. However, by looking at this diversity, we found that base-pairing between tracrRNA and the crRNA and the interaction with Cas9 protein was highly conserved. So very quickly we synthesized the RNAs and the proteins of different bacterial species, knocked out all the important domains, etc., and ultimately found the mechanism targeted the DNA and not the RNA.

Jennifer explained a little bit about the principles of how the system works. What is important to mention is that it is really a unique system, and very flexible in the case of Streptococcus pyogenes, and this is where maybe we were very lucky in my laboratory to work on this bacterium. As I said, the system exists in a number of bacterial species. We and others have tried to adapt this mechanism not only from Streptococcus pyogenes but also from different bacterial species in order to apply the CRISPR-Cas9 technology to target DNA in diverse cells and organisms. And it seems that the system from Streptococcus pyogenes works particularly well, thanks to the flexibility of this protein, which undergoes specific conformational rearrangement each time that the protein first binds to the RNA molecules, and then recognizes the DNA, and then cleaves the DNA. Something that I would like to mention is that we always speak about protein guided by two RNA molecules forming a duplex. Jennifer explained the idea of how we simplified the system by adding a linker that would allow the use of a single RNA instead of two RNAs, which can be useful in certain types of applications. There is another component that is important for the system to work, which is a sequence in the DNA that is called the protospacer 


\section{Potential applications of the CRISPR-Cas9 system}

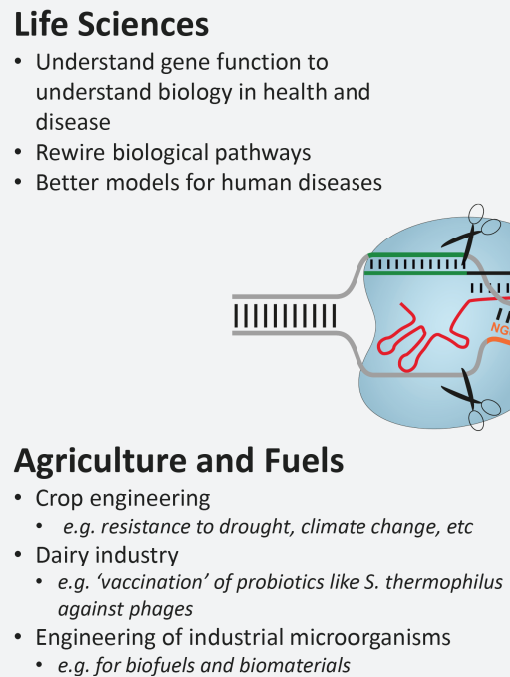

- Understand gene function to understand biology in health and disease

- Rewire biological pathways

- Better models for human diseases

Agriculture and Fuels

- Crop engineering

- e.g. resistance to drought, climate change, etc

- Dairy industry

- e.g. 'vaccination' of probiotics like S. thermophilus against phages

- Engineering of industrial microorganisms

- e.g. for biofuels and biomaterials

on these technologies to show that they could apply the technology for their own purposes to have a precise genetic tool for genome engineering. So, everyone agrees that the technology offers a number of advantages: it's cheap and easy, it's very efficient, it's versatile, and it allows multiplexing in the sense that one can design different RNA molecules to simultaneously target different sequences in the genome. The system naturally is already highly specific, and a number of improvements have been made to make it even more specific. It is important for many applications of the technology that you would like to modify a DNA sequence of interest and not any other sequences in the genome. The adjacent motif (PAM). It is a short stretch of nucleotides NGG in the case of Streptococcus pyogenes. This motif has to be present on the target DNA for the Cas9 system to work. And the work of Sylvain Moineau, who published a paper in Nature in 2010, was really critical to come to the conclusion that this motif is important for CRISPR-Cas9 system to cleave DNA and introduce a double-stranded DNA break. Except that at the time tracrRNA was not known, so the exact mechanism was not really known.

When we saw how the enzyme was working, and we ultimately showed that in a very simple manner by just exchanging a portion of this RNA molecule, one could finally bring the system to an RNA-programmable technology. It was clear as it was working so well in the test tube, that a very efficient enzyme from Streptococcus pyogenes could offer considerable potential for genome editing in cells in the six kingdoms of life for biotechnological, biomedical and gene therapeutic purposes. And indeed, as I said, already six months, a little bit more than six months after we published with Jennifer the paper in Science, a number of scientists showed in publications from January to March 2013 that the technology was working very well to perform genome editing and engineering in human cells, as well as in mice, bacteria, plants, in other model organisms, and also in monkeys and organoids. I would like to mention those from the scientific community in the Boston area who were working early on in human cells were the groups of Feng Zhang and George Church and very quickly actually the entire world picked up system has low toxicity, and there are a lot of efforts from the scientific community to develop delivery systems to bring the technology into the right cell or tissue type. You can correct mutations, you can delete portions of genes, you can add new sequences at the site of interest, you can replace a certain gene or a certain sequence by another sequence of interest. And as I said, the technology is really useful and works in any type of cell or organism.

These are just some aspects of the Cas9 genetic toolbox. What is really fascinating about this enzyme is that it has, by its nature, domains that allow it to cleave DNA, but you can also easily mutate these domains. And this was shown also by the group of Šikšnys. You can convert the enzyme that cleaves DNA into an RNA-programmable protein that can bind DNA but does not cleave DNA. And then you can fuse this RNA-programmable Cas9 to different domains that allow you to, for example, activate gene expression or inhibit gene expression. There have been a lot of screens that have been developed using this aspect of the technology. You can also fuse the Cas9 protein to effector domains that allow you to modify DNA and to study epigenetic phenomena. You can also mark the DNA. So you can do many different tricks with Cas9. It has many applications for example in human medicine and synthetic biology, and in agriculture for the production of new crops.

I would like to finish by mentioning the aspect of human medicine. The CRISPR-Cas9 technology has both indirect and direct implications here. As an indirect application, it allows 


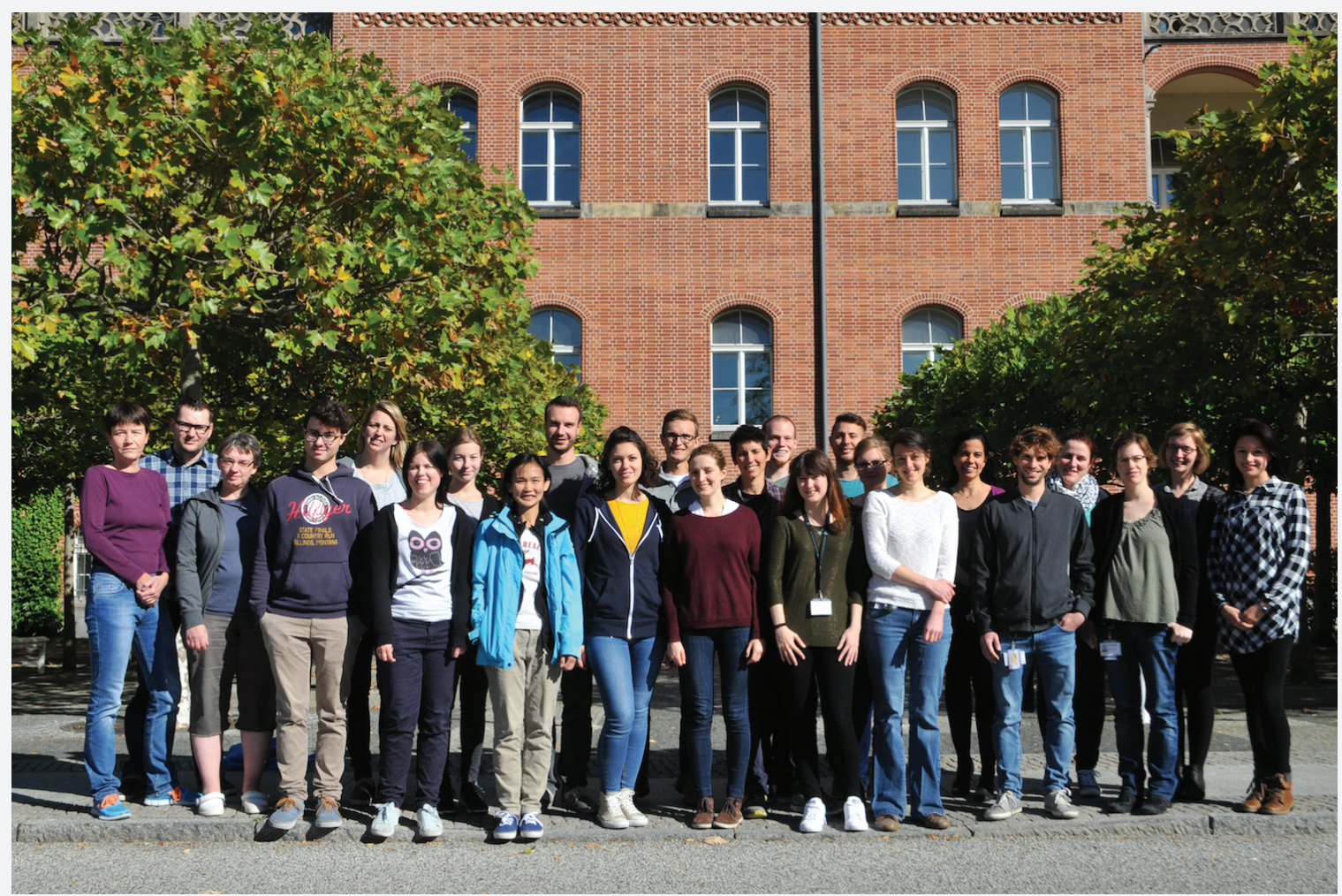

The Charpentier Lab in Berlin, Germany (October 2016).

us to work with the right type of human cells. We were always a little bit restricted - specifically in my field of research, infection biology - in always having to work with the same types of human cell lines that are not always the best cells to work with but are the easiest to genetically manipulate. With CRISPR-Cas9, you can readily perform genetics in more types of cells. Having the right technology allows us to understand functions of genes and unravel new pathways, and also to engineer new disease models, and to humanize disease models in order to better understand diseases. A lot of effort has been undertaken by the community to develop new types of screens for therapeutic targets, and ways to validate these targets. And the bed-to-bench approach - that is using patient genome sequences to investigate genetic predisposition for certain diseases - allows us to go back to the bench and really test if the mutation may be cause the disease. The big challenge though is to develop the technology as a direct therapeutic for genetic diseases, which is specifically a field I am interested in. So, what I want to say is that this technology is really helpful not only in basic science, but also in biotechnology and in the pharmaceutical industry. In human medicine, it allows us to study the diversity of the world, which is really important because this can allow us to challenge certain dogmas or to make breakthroughs in our understanding of biology. We continue working on different types of mechanisms in my lab, focusing on infectious diseases, and hoping that CRISPR-Cas9 will be very helpful.

I would like to finish with some acknowledgments. Indeed, research nowadays is quite exciting because you have the possibility to really travel around the world. This has been very important for me. This research developed in Austria, Sweden and Germany. And prior to Austria, Sweden and Germany, I was working in the US and France. I really encourage you to travel because you can appreciate different types of cultures and different ways approaching science. You have the opportunity to meet great scientists. Sometimes you do not realize that they may have affected your life or inspired you until much later. In science, you also need to always find potentially new aspects of topics, and to see things from a different perspective. Moving to another country and getting to know various types of administration and funding organizations is a challenge in itself, and it is good to challenge yourself. So, I would like to thank organisations and institutions in Austria and Germany, but specifically in Sweden. The idea to focus on CRISPR and bring those two topics - tracrRNA and CRISPR — together really came in Sweden at the Laboratory 
for Molecular Infection Medicine Sweden at Umeå University. I would like to thank a wonderful team of scientists, since without talented young researchers nothing would happen. I would like to specifically mention Elitza Deltcheva, and $\mathrm{Kr}$ zysztof Chylinski, and others. It has always been very inspiring to work with young scientists who are enthusiastic and bring new and refreshing ideas. And my collaborators, a group including Jörg Vogel in Würzburg, Jennifer Doudna and Martin Jinek, and Eugene Koonin for studying the evolution of the system. This picture shows my lab when there were summer students last year. I would like to mention ERS Genomics and CRISPR Therapeutics, two companies I co-founded, and also for full disclosure of any conflict of interest.

And I will finish with some quotes. One was from Louis Pasteur, over 150 years ago. "In the field of observation, chance favours the prepared mind." I think it is very true. "Let me tell you the secret that has led me to my goal. My strength lies solely in my tenacity." And I think this is important for science. "Persistence in scientific research leads to what I call instinct for truth." And this is also important. I like also this one from François Jacob. It is about night science, and it reminds me of my time in Umeå. "Night science: a stumbling, wandering exploration of the natural world that relies on intuition as much as it does on the cold, orderly logic of 'day science'. In today's vastly expanded scientific enterprise, obsessed with impact factors and competition, we will need much more night science to unveil the many mysteries that remain about the workings of organisms." And at least for my part, Sweden has been great. You go to the North, no one cares about whether you are alive or not. You can be quiet and you can focus and it is great. 